\title{
Identifying Risk at the Conceptual Product Design Phase: A Web-Based Software Solution and Its Evaluation
}

\author{
Katie Grantham, Cassandra Elrod, Barry Flaschbart, William Kehr \\ Missouri University of Science and Technology, Rolla, USA \\ Email: kag@mst.edu
}

Received January 31, 2012; revised February 24, 2012; accepted March 5, 2012

\begin{abstract}
This paper introduces a web-based application that enables engineers or designers to identify risks during the conceptual phase of a product design. This software application was developed based on the Risk in Early Design (RED) method developed by Author, Stone, and Tumer. The RED method demonstrates that risks can be identified in the early phase of product design by relating recorded historical failure information to product functions. Based on the concept of RED theory and current industry needs, the goals of a web-based RED application were defined. These goals are the intended benefits or functionalities that the web-based RED application would provide. In addition, a multi-level evaluation framework was adopted to determine how well the application meets the needs of various organizations. As part of the evaluation, a questionnaire was developed and administered to a sample industrial and academic user group. The results of the evaluation indicate that the web-based RED software application meets many of the goals to help an organization in performing product risk analysis.
\end{abstract}

Keywords: Product Design; Design Management; Risk Management; Risk Modeling

\section{Introduction}

The purpose of this paper is to present a knowledge based conceptual design software tool (the RED software application) to promote failure free design and to demonstrate its effectiveness through an evaluation. A knowledge base of recorded historical information is vital in preventing past failures of engineering products from recurring in future designs. Risk in Early Design (RED) is a knowledge based probabilistic risk assessment method that leverages historical failures to identify and quantify product risks even in their conceptual stage of development. The early availability of risk information allows improvements to be made to the design at a stage when the penalty for change is minimal. RED also prevents the need for having expert teams using expensive resources for risk identification and enables them to focus on risk mitigation, a better use of their expertise. This paper describes a web-based software solution to enable RED theory to be utilized by a multitude of entities for early risk identification and prevention. Further, the paper also presents an evaluation schema and a preliminary industrial evaluation to ensure the software solution meets the needs of it target users.

Current industry risk assessment tools require experts to identify potential failures of a product [1-8]. Expertdriven analysis, though valuable, is not flawless because of personal biases, cost and time constraints [9]. The RED method and subsequent software application was developed to overcome these issues. This application plays a vital role between the product designers and failure prevention. RED leverages design knowledge such as product functions and components and links them through a knowledge base to historical failure reports. This unique linkage enables even inexperienced designers or engineers to easily perform a risk analysis during the conceptual phase of product design.

The goal was to provide a software solution based on the RED concept and to verify that the solution met all the requirements of practicing industry professionals. Before releasing software for any institutional use, it must be verified that it meets the needs of an organization. Further, within the engineering community, software systems have a reputation for being undependable, especially in the first few years of their use [10]. For these reasons, a multi-level evaluation framework that is used for software system evaluation was adopted. It provides options for defining elements such as goals, evaluation objectives, metrics, and measures that are conceptual and implementation-specific [11]. The conceptual measures identify the type of data to be collected and the implementation-specific measures identify the specifics of a particular collection instance, for example, data element, collection tool, collection method [11]. This multi-level evaluation framework guided the development of the software application. A questionnaire was used as an 
evaluation tool to gather users' feedback on the RED software application. Finally, the feedback results from users were analyzed to verify that the goals of the software application have been met.

\section{Background}

\subsection{Risk Analysis Software}

Current risk software available generally involves largescale automation of the systems, in the form of enterprise-wide risk management systems. These software systems also regularly use several techniques for prediction, estimation and risk diagnosis. The RAP (Risk Analysis Prototype) system was developed internally by the Jet Propulsion Laboratory for space exploration missions. It was designed for specially trained project teams and requires input from technical specialty areas. It considers risk throughout the design process, emphasizing communication among experts. Resources required are considerable, including other specialty software applications, input from subsystem experts, and a complex communication architecture [2].

IRMAS (Intelligent Risk Mapping and Assessment System) was developed through a collaboration between business and academic experts and is not available to the general public. IRMAS appears to be industry independent, seeking to manage both the project life cycle and associated risks. It is a complex system, with wide data collection needs, designed for use by project managers as opposed to persons active in the early conceptual phases of new product development. The sytem requires input from many organizational levels, addressing eight different areas of risk, and can capture and reuse lessons learned from previous projects [12].

Certus is a commercially available software system that can be purchased from Neohapsis, Inc., which also provides product training. Major customers include the federal government along with companies in the following industries: financial services, healthcare, insurance, manufacturing, and power/energy. Certus is part of Neohapsis's risk consulting business and training; implementation of the software includes training, planning, and assessment activities. The software is offered as a single system to minimize complexity. Aimed at companywide day-to-day operations, Certus consolidates risk management and a variety of compliance issues [13].

Designsafe is used in a wide range of industries; however, packing machinery and robotics associations were instrumental in creating the software. Potential users can download a free demo. Training is available on DVD for $\$ 325$. Designsafe improves product designs by letting design engineers complete risk/hazard assessment for their products and proesses while also allowing ongoing assessments and scenario investigations. The system is customizable, and includes networking and library and file sharing [4].

CORA is a software system available for purchase from All Hazards Management, a company that concentrates on emergency preparedness. CORA is one of three major software systems available; the other two deal with information management and assessment and emergency response management; CORA deals with costs. The system can recycle the efforts of risk experts whle using field personnel to collect data. It is primarily aimed at on-going operations threats and planning for emergencies; it demonstrates the financial impacts of risks, and allows scenarios [7].

CHAMPS, Inc. began as a nuclear power plant construction and startup engineering firm. The CHAMPS product is a commercial software system. Customers listed on the company's web site include four electric power generation/distribution companies and Quaker Oats. The system is advertised as an enterprise asset management, customer relationship management, and business intelligence tool. CHAMPS offers free on-line product training seminars [8].

\subsection{Software Evaluation Methods}

Organizations often procure and use software without considering any sort of evaluation of that software [11]. When software systems are considered for institutional use, it is critical to determine the effects the software will have on the organization. During software product evaluation, the ability of the software product to meet the user's needs of that product are determined [14]. Rombach states that in order for measurement to be successful, effective "top-down" strategies that derive metrics and associated measures from goals and interpret measurement data in the context of goals are needed [15]. Approaches using a "top-down" manner of identifying useful metrics from goals include the Software Quality Metrics (SQM) approach by Murine [16], which is based on prior work by Boehm [17] and McCall [18], the Quality Function Deployment (QFD) approach by Kogure and Akao [19], and the Goal/Question/Metric (GQM) approach by Basili $[20,21]$.

SQM is a rigorous, precise software quality methodology consisting of measurable quality factors, criteria, and elements [13]. The objective of SQM is to produce cost-effective quality software. The SQM measurement approach includes three key elements, namely factors, criteria, and metrics. Important Quality Factors (such as correctness, reliability, etc.) are chosen from twelve factors, which include one added to McCall's 11 Factors. Each Quality Factor is further defined by set of attributes called criteria. For example, "consistency" and "accuracy" of the software are some of the example criteria for the factor "reliability". Each criteria is quantified by indi- 
vidual measurements, metrics, and they are decided in response to the necessity for each criteria chosen.

QFD is a method for developing a design quality aimed at satisfying the consumer and then translating the consumers' demands into design targets and major quality assurance points to be used throughout the production stage [4]. In planning a new product, QFD starts with the customer requirements, which are identified and defined through market research. This is called a product development process, which includes program planning, conceptualization, optimization, development, prototyping, testing, and manufacturing functions. The development process is very complex and often cannot be performed by one individual. It is precisely the complexity of QFD that all too often causes the product development process to create a product that fails to meet the customer requirements.

The GQM approach is based upon the assumption that for an organization to measure in a purposeful way it must first specify the goals for itself and its projects, then it must trace those goals to the data that are intended to define those goals operationally, and finally provide a framework for interpreting the data with respect to the stated goals [7]. Thus it is important to clearly understand what informational needs the organization has, so that these needs for information can be quantified and the quantified information can then be analyzed as to whether or not the goals are achieved. The GQM approach provides the three key elements embodied in its name: Goal, Question, Metrics. At a conceptual level, a goal is defined for an object, for a variety of reasons, with respect to various models of quality, from various points of view, relative to a particular environment [7]. At an operational level, a set of questions is used to characterize the way the assessment or achievement of a specific goal is going to be performed based on some characterizing model [7]. At the quantitative level, a set of data is associated with every question in order to answer it in a quantitative way [7].

On comparing the three approaches, SQM and QFD are limited to product quality, whereas GQM can be used for process as well as product quality [22]. QFD is a complex and long process. The GQM approach is a systematic way to tailor and integrate an organization's objectives into measurement goals and refine them into measurable values [23]. GQM was developed for use with software improvement projects, and it is used in a majority of research and development software projects [11]. The GQM paradigm prescribes setting goals in operational and tractable ways where goals are refined into a set of quantifiable questions that specify metrics. Data are tied to specific metrics that, in turn, are tied to specific goals [11]. An example to show how goal, question and metrics can be tied based on GQM is presented be- low. The GQM method was used to identify the most appropriate metrics for assessing the software inspection process conducted by AT\&T developers [24].

Goal: Monitor and control.

Question: What is the quality of inspected software?

Metrics: Avg. faults per KLOC, Avg inspection rate.

Note: KLOC (thousands of lines of code) is a traditional measure of software size; it is often related via empirical formulas to or how long or how many people it will take to complete a software development project.

The above metrics are defined for the technical or algorithm level software evaluation. Technical or algorithm level evaluation determines the quality of a product. RED evaluation primarily focuses on organizational needs and to ensure if the needs are fulfilled via the software solution. Therefore, technical or algorithm level evaluation was not a major concern in the web-based RED software evaluation. Further, industry feedback about the webbased RED software application will be taken into consideration; performing a quality evaluation will be meaningful at that time. Since SQM and QFD primarily focus on quality, therefore, they were not chosen for the web-based RED software evaluation. GQM is one of the most widely used methods of its class; it focuses on process in addition to quality. After consideration, though, none of these methods were used directly. The software evaluation did, however, recognize the benefits of the GQM methodology and its multi-level evaluation framework. The objective of the web-based RED software evaluation in this paper is not to perform technical or algorithm level evaluation but to provide a way to decide and define "what to measure" and "how to measure" the software solution in order to conclude that the organizational needs are met. Therefore, the multi-level framework was adopted, which provides measures that differentiate conceptual elements from implementation-specific elements that GQM does not provide [11]. These elements are considered as further refinements to the GQM methodology. Here, the conceptual measure helps to identify the type of data to be collected (what to measure) and the implementation-specific measure helps to identify the collection method (how to measure). The data collection results conclude whether or not the goals of the solution are met.

\section{Risk in Early Design (RED) Software}

\subsection{RED Theory}

The Risk in Early Design (RED) [25] theory mathematically maps product functions and failures to likelihood and consequence elements based on cataloged historical failure information. Equation (1) is used to obtain the function-failure mode matrix of the RED theory using cataloged historical failure modes. 


$$
\mathrm{EC} \times \mathrm{CF}=\mathrm{EF}
$$

The matrices in Equation (1) are the function-component matrix (EC), the component-failure matrix (CF), and the function-failure matrix (EF). These are the critical matrices that make up the knowledgebase that the software performs risk calculations on.

RED uses matrix-based risk assessment to easily identify specific function-failure mode combinations that have historically occurred and to quantify that information into consequence and likelihood based on the outcome of the historical events [25]. Because this method uses documented historical function-failure combinations, even novices will be able to use it to determine potential risks in the products. Also, RED's historical foundation helps to remove personal bias [25]. RED can be applied even in the conceptual phases of product design. Avoiding risks at this stage is less costly because the product's physical form has not yet been determined.

Communication of the risks generated by RED is manipulated so that the risks are compatible with the risk fever chart [25]. The risk fever chart was originated by Defense Acquisition University (DAU) [26]. This chart consists of a plot of consequence versus likelihood of risks. The RED method is mapped such that both conesquence and likelihood have integer values ranging from one to five.

The categories in the RED heuristics are: system level, subsystem level, human centric, and unmanned. System Level is a design state that considers the product as a whole; Subsystem Level is a design stage that considers subsystems or smaller pieces of the product; Human centric is a product that requires human input for its operation; and unmanned product is a product that does not require direct human interaction during operation.

Author et al. [25] illustrated a RED risk assessment performed on a functional model of a Thermal Control Subsystem. From the functional model of the Thermal Control Subsystem the functions from the functional basis were collected and used to select entries of the function-component (EC) matrix. Using the EC matrix thus formed, the product-specific function failure (EFprod) matrix was determined. Next, the risk likelihood and consequence calculationswere performed using the functioncomponent (EC) matrix and component-failure severity (CF') matrix. The sample of risks identified from the RED risk assessments performed on the thermal control subsystem risk assessments are shown in Table 1. A software application to perform these risk calculations will greatly improve the usability of the RED method and the reliability of electro-mechanical products.

\subsection{RED Software Goals}

The evaluation in this paper is aimed at establishing the
Table 1. Sample risks from thermal control subsystem RED risk assessments.

\begin{tabular}{lc}
\hline Risk Element Statement & $(\mathrm{C}, \mathrm{L})$ \\
\hline Export thermal energy fails due to high cycle fatigue & $(5,5)$ \\
Export thermal energy fails due to yielding & $(4,3)$ \\
Guide gas fails due to thermal fatigue & $(5,2)$ \\
\hline
\end{tabular}

RED software application's effectiveness, that is, how well the RED software application fits the needs of an organization with respect to product risk analysis during conceptual design. Looking at organizational factors affecting acceptance of new technology, Meyer [27] lists 4 factors that are important in achieving implementation of a new technology. First are the expectations of the organization and employees; how the product performs in relation to what they were lead to believe, thought or hoped for. Second is the performance of the innovation, in this case the RED application. Third is conflict within the organization, not dissimilar to the situation discussed above. Finally is the extent of adaptation to the existing organization that is needed when bringing in the new technology or, in this case, software.

With all these considerations, a generic software solution is developed to perform risk analysis of any Industrial product. This provides features for the users to perform risk analysis for any product as long as it complies to the RED input data format. Hence, a careful and thoughtful consideration is given to the choice of evaluation framework to define the goals of the RED software application, wherein these goals are not biased to any specific industry (i.e. they are industry-independent goals). The web-based RED application leverages the multilevel evaluation framework developed by Jean Scholtz and Michelle Potts Steves from the National institute of Standards and Technology, USA [11]. This is a multilevel framework that helps to structure evaluations by mapping system goals to evaluation objectives, metrics, and measures. Five goals of the RED software application were defined as follows as part the development framework. Along with the listing of these goals, their relationship to the state-of-the art (RSOA) in available risk assessment tools is provided.

Goal 1: To provide a widely accessible risk assessment tool.

RSOA: The tools described in Section 2.1 are either unavailable to the public or must be purchased.

Goal 2: To provide an independent risk assessment tool.

RSOA: While some of the systems discussed were not closely tied to any industry, most had a somewhat narrow applicability.

Goal 3: To provide a risk assessment tool that enables even inexperienced engineers to assess risk. 
RSOA: The complexity of the commercially available systems is a major drawback. Further, the reliance on experts to identify the risks prior to analysis is also an inhibitor for designers.

Goal 4: To provide a risk assessment tool that can be used as early as the conceptual phase of product design.

RSOA: The systems identified in Section 2.1 require detailed product designs as minimum to perform and/or track product risks.

Goal 5: To provide a tool that reduces resources required to perform a risk analysis.

RSOA: The complexity and cost of the available risk assessment tools do little or nothing to reduce the manhours or machine power to assess risks.

These goals were used to design the web-based RED software as described in Section 3.3.

\subsection{RED Software Design Features}

The first goal of the web-based RED application was to provide a widely accessible risk assessment tool. By its nature, a web-based solution satisfies this need better than an application to be installed and run in each situation. Further, a web-based application can be accessed from anywhere, with the help of the Internet. The second goal of the RED application focuses on providing a risk assessment tool that can be used by many industries. To achieve this goal, the application provides options for performing risk analysis based on user-defined data. User-defined data are the historical failure reports from the industry. The only requirement is that they must adhere to the RED matrix data format. Uploading options on the web-based application fulfill the goal. As a result, any industry can upload their product historical failure reports to perform risk analysis. The third goal was to provide a tool that enables even inexperienced engineers to assess risk. Hence the application's ease of use and expressive capability were major concerns here. Expressive capability is the presence or absence of various options in the application. Therefore, the application had to be devel- oped with all the basic functions and capabilities that an inexperienced user would expect it to have. In addition, to avoid complexity, the solution must help the user to accomplish the risk analysis task in a few steps as well as be easy to learn and understand. The fourth goal was to provide a risk assessment tool that could be used as early as the conceptual phase of product design. The application provides results with essential information that enables users to make better decisions during the early phase of product design. The fifth goal of the RED application was to provide a risk assessment tool that reduces resources required to perform the analysis. Therefore, the application is designed to reduce cost in terms of time and personnel. Table 2 provides a mapping that shows how specific design features of the software support the goals.

\subsubsection{Web-Accessibility}

A free web-based solution was chosen as the design feature to provide a widely accessible risk assessment tool. Access to a knowledgebase of electromechanical product failures enables product designers to focus on risk mitigation during their design process rather than failure research. Also, internet accessibility allows the RED software users to access the most up to date knowledgebase and perform risk assessments without the need for installing software, checking computer compatibility, or checking for updates to the failure knowledgebase.

\subsubsection{Historical Failure Knowledgebase}

As stated previously, RED enables risk identification with the use of a knowledgebase of historical product failures. The derived engineering languages for functions [28], components [29], and failures [30] are the backbone for effective construction of a RED knowledgebase. Previous research efforts focused on the construction of the electromechancial product failure knowledgebase [31] which is available for use in the RED program. The failure reports currently cataloged in the knowledgebase come from National Transportation and Safety Board

Table 2. Goal based RED software design features.

\begin{tabular}{|c|c|}
\hline Goal & Supporting Design Feature \\
\hline 1 To provide a widely accessible risk assessment tool & - Web-accessibility \\
\hline 2 To provide an industry independent risk assessment tool & - $\quad$ User-defined data upload \\
\hline $\begin{array}{l}3 \text { To provide a risk assessment tool that enables even inexpe- } \\
\text { rienced engineers/product designers to assess risk }\end{array}$ & $\begin{array}{ll}\text { - } & \text { Historical failure knowledge base } \\
\text { - } & \text { Minimal product knowledge requirement } \\
\text { Specific risk output }\end{array}$ \\
\hline $\begin{array}{l}4 \text { To provide a risk assessment tool that can be used as early as } \\
\text { the conceptual phase of product design }\end{array}$ & - $\quad$ Minimal product knowledge requirement \\
\hline $\begin{array}{l}5 \text { To provide a risk assessment tool that reduces resources } \\
\text { required to perform a risk analysis }\end{array}$ & $\begin{array}{l}\text { - } \quad \text { Historical failure knowledge base } \\
\text { - } \quad \text { Minimal product knowledge requirement }\end{array}$ \\
\hline
\end{tabular}


(NTSB) reports, consumer product failure reports, ASM handbooks, etc. The benefit of having a historical knowledgebase of product failure available from which to identify risks allows even novice product designers "experience" that allows them to make critical design decisions early in the process thus saving time, resources, and eventually consumers from harm.

\subsubsection{User-Defined Data Upload}

Since risk exists in all domains, it is not sufficient to simply be able to calculate the risk of electromechanical products. The general equations for RED can be applied to other domains for assessing risk [25]. All that is necessary for the risk domain change is an appropriate database of historical failures. For example, Vucovich, et al. [32], used RED theory to calculate the risks of software products. Furthermore, Patil, et al. [33] have used RED to perform risk assessments in the business domain, and Author et al. [31] have leveraged the RED theory to predict risks for the implementation of lean manufacturing products [34] as well. Therefore, the web-based RED software includes a feature that enables users to upload their own database (or user-defined data) to assist in their risk assessments.

User-defined data are historical failure reports that must adhere to the RED matrix data format. Uploading options on this web-based application allow this capability and fulfill the goal. As a result, any industry can upload their product historical failure reports to perform risk analysis should they want to use their own electromechanical product database or analyze risk in a different domain. If the user-defined input data fails to meet the formatting requirements, then the user will be prompted with error messages. The messages were designed to indicate the type of error, in order to ease upload for the user. For example, matrix dimensional errors and file type errors are called out to the user.

\subsubsection{Minimal Product Knowledge Requirement}

The product-specific knowledge required to perform a risk analysis with RED is minimal. This feature primarily allows risk analyses to be performed earlier in the design process, an important quality not found in other available risk assessment software. The product-specific knowledge required to use RED includes product functionality, product type (human-centric or unmanned), and the design level of risk analysis desired (sub-system or system level). The first two required areas of product knowledge can be derived directly from customer needs that often initiate the design process for new products [28]. The final product knowledge requirement is most often internal to the organization performing the risk analysis. For example, if the risk analysis is being performed on the drive train of an automobile, the subsystem risk analysis would be selected by the RED user to identify the most risky areas in the drive train. However, if the user desires to perform a risk analysis on the entire vehicle, perhaps to determine its feasibility to continue through the design process, that user would select a system level risk analysis. Though detail design criteria such as material selection, performance parameters, etc. are not needed to initiate a risk assessment through RED, it is intended that the user will incorporate this information to update the risk analysis as it becomes available.

\subsubsection{Specific Risk Output}

The RED software application outputs the risk results in two forms. The first is a Fever Chart [26] where the green area represents low risk, the yellow area represents moderate risk, and the red area represents high risk. The chart shows consequence on the horizontal axis and likelihood on the vertical axis. The number displayed in the color-coded area represents the number of risk elements with that likelihood and consequence value. This common graphical representation of risk enables users to quickly gage the risk status of their system.

The second type of risk output is a text file featuring specific risk statements. The risk statement provides a clear, concise, and informative message so a user easily understands the risk. Every risk statement is displayed in a the following format:

"Function fails due to Failure Mode at (Consequence Value, Likelihood Value)”.

The risk statement file groups the risk statements into the high, moderate, and low categories and is formatted so it is compatible with spreadsheet software.

The application, as developed, provides a menu with instructions for the user. The instructions take the form of a series of steps, with "clickable" selections to carry out appropriate actions. The instructions/steps are listed below:

Step 1: Engineers must prepare functions of the product design that are to undergo risk analysis.

Step 2: Start the RED application.

Step 3: If the risk analysis is for an electromechanical product, the designer may choose the S\&T database to perform risk analysis.

(Note: The S\&T database supports electromechanical products only. If not an electromechanical product, the user can upload user-defined data that is populated based on historical product failure information. User-defined data must comply with RED matrix dimension standards.)

Step 4: If the S\&T database is the choice for performing risk analysis, then go to Step 9; else, go to Step 5.

Step 5: If user-defined is desired, then upload three input matrices (as Excel .xls files), Function Component matrix, Component Failure matrix, and Component Failure Severity matrix. 
Step 6: After uploading user-defined data, it confirms file upload the matrix dimension.

Step 7: If the verification status is successful, then go to step 8; otherwise, go to step 5 .

(Note: Verification may fail due to a wrong input file type or if the input data does not comply with the RED input data format.)

Step 8: User-defined data is now available to undergo risk analysis.

Step 9: The RED application displays product functions and the user can choose the desired product functions that must undergo risk analysis.

Step 10: The user must now choose the appropriate design level and product type for the product under risk analysis.

Step 11: The application displays the fever chart and generates a risk file to be downloaded by the user.

Step 12: The user can review the risk statement to help make decisions about the product design.

Step 13: Termination of the RED application.

\section{RED Software Evaluation}

After the development of the web-based RED application, the next step was to ensure that the goals defined in the evaluation framework were met. To perform this assessment, a questionnaire-based evaluation model was adopted. The questions were framed based on the goals defined in the RED evaluation framework. User responses to these questions help determine whether the web-based RED application meets the defined goals.

\subsection{Software Evalutaion Metrics}

The evaluation framework consists of five elements or levels: system goals, evaluation objectives, conceptual metrics, and measures, both conceptual and implementation-specific. The framework element system goal is the intended benefit or functionality the software system will provide. This goal may reflect a technical performance goal for the software system or an organizational goal. The next level of framework is the evaluation objective level. Each objective drives a particular part of an evaluation, within the context of its associated system goal. Evaluation objectives (EO) partition evaluation concerns in the overall assessment of a particular goal. A metric is the interpretation of one or more contributing elements, examples, measures or other metrics, corresponding to the degree to which the set of attribute elements affects its quality. A measure is an observable value, while a metric associates meaning to that value by applying human judgment, often through a formula based on weighted values using the contributing measures. Each evaluation goal will have one or more conceptual metrics. Metrics, when assessed with their associated measures, will contribute to an assessment of how well a particular system goal was met. The lowest two levels in the framework constitute measures: conceptual and implementation-specific. Conceptual measures identify the type of data to be collected. Implementation-specific measures identify the specifics about a particular collection instance, for example, data element(s), associated tool, collection method [11].

\subsection{Evaluation Questionaire Development}

Each goal has one evaluation objective and every evaluation objective has at least one metric defined. Every metric, in turn, has its own measures wherein user ratings are considered and has an implementation-specific measure in every goal. Thus, a questionnaire was considered as an effective tool for collecting user ratings. Questions are mapped to the appropriate goals.

The questionnaire was designed to measure the goals using the evaluation metrics presented in Section 4.1. Two set of questionnaires were given to the students- $\mathrm{a}$ User Evaluation Questionnaire and a RED Tool Evaluation Questionnaire.

User Evaluation Questionnaire-Questions were framed with an aim of understanding the user experiences and knowledge about Risk analysis concepts and tools. These questions were designed to assist in the understanding of the skill levels in the sample population.

RED Tool Evaluation Questionnaire-These questions were framed in order to enable mapping to the goals of the web-based RED application. Questions found in the questionnaire were actually framed based on the RED solution goals. Table 3 shows how questions were mapped to the goals of the RED solution. For easy reference, only the goal and question numbers are listed in the table. For example, Questions 1 and 2 of the RED tool evaluation questionnaire were mapped to goal 1: "To provide a widely accessible risk assessment tool”. The respondents were asked to rate each question on a 5-point Likert scale of 1 to 5 , where 1 represents strong disagreement (which is the least user satisfaction rating) and 5 represents strong agreement (which is the maximum user satisfaction rating for any question).

\section{Evaluation Results}

Ideally, the evaluation would be performed by a large and diverse group of engineers. An initial smaller group was utilized in order to obtain rapid feedback. Additional evaluations need to be pursued in the future.

\subsection{Respondent Knoledge Level}

The RED tool was evaluated by students (users) at Missouri University of Science and Technology, Rolla, Missouri (Missouri S \& T). Six students who were enrolled 
Table 3. RED tool evaluation question mapped to goals.

\begin{tabular}{|c|c|c|c|c|c|}
\hline Goal & $\begin{array}{l}\text { Evaluation } \\
\text { Objective }\end{array}$ & Metric & Conceptual Metric & $\begin{array}{l}\text { Implementation } \\
\text { Specific Metric }\end{array}$ & $\begin{array}{l}\text { Survey } \\
\text { Question }\end{array}$ \\
\hline \multirow{2}{*}{$\begin{array}{l}\text { 1. To provide a widely accessible risk } \\
\text { assessment tool }\end{array}$} & \multirow{2}{*}{$\begin{array}{l}\text { Assess solution for } \\
\text { geographic } \\
\text { accessibility }\end{array}$} & \multirow[t]{2}{*}{ Accessibility } & $\begin{array}{l}\text { Geographically diverse } \\
\text { user capability }\end{array}$ & \multirow{7}{*}{ User/designer rating } & 1 \\
\hline & & & Multiple user capability & & 2 \\
\hline $\begin{array}{l}\text { 2. To provide an industry independent } \\
\text { risk assessment tool }\end{array}$ & $\begin{array}{l}\text { Assess solution for } \\
\text { independence }\end{array}$ & Adaptability & $\begin{array}{l}\text { Capability of application } \\
\text { to be used by different } \\
\text { industries }\end{array}$ & & 3 \\
\hline \multirow{3}{*}{$\begin{array}{l}\text { 3. To provide a tool that enables even } \\
\text { inexperienced engineers to assess risk }\end{array}$} & \multirow{3}{*}{$\begin{array}{l}\text { Assess intuitiveness } \\
\text { of application }\end{array}$} & \multirow{2}{*}{$\begin{array}{l}\text { User satisfac- } \\
\text { tion with Inter- } \\
\text { face }\end{array}$} & Ease of use & & $4,5,6,7$ \\
\hline & & & Expressive capability & & 8 \\
\hline & & Knowledge & Amount of information & & 12 \\
\hline $\begin{array}{l}\text { 4. To provide a risk assessment tool that } \\
\text { can be used as early as the conceptual } \\
\text { phase of product design }\end{array}$ & $\begin{array}{l}\text { Assess early risk } \\
\text { information }\end{array}$ & Result accuracy & $\begin{array}{l}\text { Application result } \\
\text { reliability }\end{array}$ & & 9 \\
\hline $\begin{array}{l}\text { 5. To provide a risk assessment tool } \\
\text { that reduces resources required to } \\
\text { perform the analysis }\end{array}$ & $\begin{array}{l}\text { Assess total resources } \\
\text { required for risk } \\
\text { assessment }\end{array}$ & Economical & $\begin{array}{l}\text { Time cost } \\
\text { Personnel cost }\end{array}$ & $\begin{array}{l}\text { User ratings/determined } \\
\text { in a real world sce- } \\
\text { nario and data }\end{array}$ & 10 \\
\hline
\end{tabular}

in the Function Based Failure Analysis course offered at Missouri S \& T were considered as respondents for this evaluation. This course provides a solid knowledge of Risk analysis techniques or methods on any product. The students were graduate students who were employed full time at both Boeing and Lockheed Martin. Their range in industrial experience was 5 - 25 years. The user evaluation results for question 1 indicates that the users chosen to evaluate this RED application possess a good knowledge of product risk analysis concepts. 50\% of the users have rated themselves as having "Very Good Knowledge" and 50\% have rated themselves as "Good". Hence, these users would be ideal to evaluate the application and appreciate its value. The user evaluation results of question reveals that no experts were chosen to evaluate this application. However, $40 \%$ of the users are advanced, $40 \%$ of the users are intermediate, and $20 \%$ have no experience with other risk analysis tools. Prior to evaluating the web-based RED application, most of the sample population were given some knowledge of the application. Thus the sample population's level of experience with this RED tool ranges from expert to basic. 33\% of the users were basic, $33 \%$ were intermediate, $17 \%$ were advanced, and $17 \%$ were expert.

\subsection{RED Application Evaluation Results}

The overall sample population's responses were then verified against the goals. In evaluation of Goal 1, 67\% of the responses were "strongly agreed", 25\% "agreed" and $8 \%$ "do not know". Hence, we conclude that the application has met the goal to provide a widely accessible risk assessment tool. The evaluation of Goal 2 was also overwhelmingly positive in that the users believe that the
RED tool is industry independent with the following results: $67 \%$ of the sample population "strongly agreed", 17\% "agreed" and 17\% "do not know".

In the evaluation of Goal 3 (providing a tool that enables even inexperienced engineers to assess risk), $47 \%$ of the sample population "strongly agreed", 43\% "agreed", $7 \%$ "do not know" and 3\% "disagree". Therefore, $90 \%$ of users validated the RED applications utility for inexperienced engineers.

Goal 4's evaluation scores were the following, where $33 \%$ of the population "strongly agreed" and $67 \%$ of the sample population "agreed". In this case, the result is totally positive because the sample population had a strong understanding of RED concepts. We also saw that the sample population possesses good knowledge of product risk analysis concepts. Thus, they were able to appreciate the value of this application in assessing early risk information.

In the evaluation results of Goal 5, 52\% of the sample population "strongly agreed", $42 \%$ "agreed" and $8 \%$ "do not know". These scores indicate that the RED tool reduces resources required to perform risk assessments.

\section{Conclusions and Future Work}

The majority of user feedback is positive. These evaluation results indicate that the goals of the RED application have been met for the users who evaluated it. However, the evaluation group was small and contained only a few individuals with significant "industry" experience. Further work to have additional evaluations carried out by industry organizations is needed.

As experience in new areas is gained, additional databases like the S\&T database for electromechanical parts 
should be made available for rapid download and use, making the tool even more broadly useful.

\section{Acknowledgements}

The authors would like to thank Karthik Sundaram for the tremendous effort he put forth in coding the RED program. Also, we would like to thank Dan Nieters for his efforts in making the program available on the web.

\section{REFERENCES}

[1] L. Rosenberg, T. Hammer and A. Gallo, "Continuous Risk Management at NASA," Proceedings of Quality Week Conference, San Francisco, May 1999.

[2] L. Meshkat, S. Cornford and T. Moran, "Risk Based Decision Tool for Space Exploration Missions," Proceedings of the AIAA Space Conference, Long Beach, September 2003.

[3] Securac Incorporated. http://www.securac.com

[4] Design Safety Engineering Web Site. http://www.designsafe.com/dsesoftware.php

[5] http://www.ist-usa.com/

[6] http://www.safetyscorekeepers.com/

[7] All Hazards Management Web Site. http://www.allhazardsmanagement.com/cora.htm

[8] Champs, Incorporated Web Site. http://www.champsinc.com

[9] S. Karthik, C. Abhishek and D. Derek, "Identifying Risk at the Conceptual Phase of Product Design: A Software Solution," ASME Conference Proceedings, Vol. 2, 2007, pp. 291-298. doi:10.1115/DETC2007-35473

[10] L. Meskhat, S. Cornford, L. Voss and M. Feather, "An Integrated Approach to Risk Assessment for Concurrent Design,” IEEE Aerospace Conference, Big Sky, 5-12 March 2005, pp. 80-88. doi:10.1109/AERO.2005.1559301

[11] S. Jean and P. S. Michelle. "A Framework for Real-World Software System Evaluations," Proceedings of the 2004 ACM Conference on Computer Supported Cooperative Work, Vol. 6, No. 3, 2004, pp. 600-603. doi:10.1145/1031607.1031710

[12] B. Kayis, M. Zhou, Y. B. Khoo, A. Ahmed, R. Kusumo and A. Rispler, "IRMAS—Development of a Risk Management Tool for Collaborative Multi-Site, Multi-Partner New Product Development Projects," Journal of Manufacturing Technology Management, Vol. 18, No. 4, 2007, pp. 387-414. doi:10.1108/17410380710743770

[13] Neohapsis Web Site. http://www.riskgovernance.com/

[14] T. Punter, R. Van Solingen and J. Trienekens, "Software Product Evaluation - Current Status and Future Needs for Customers and Industry," 4th Conference on Evaluation of Information Technology, Delft, 30-31October 1997, pp. $1-11$.

[15] M. P. Steves and J. Scholtz, "Metrics Mapping Concepts," NISTIR 7061, National Institute of Standards and Tech- nology, Gaithersburg, 2003, pp. 1-12.

[16] G. E. Murine, "Applying Software Quality Metrics in the Requirements Analysis Phase of a Distributive System," Proceedings of the Minnowbrook Workshop, Blue Mountain Lake, New York, 1980.

[17] B. W. Boehm, J. R. Brown and M. Lipow, "Quantitative Evaluation of Software Quality," Proceedings of the 2nd International Conference on Software Engineering, Los Alamitos, 1976, pp. 592-605.

[18] J. A. McCall, P. K. Richards and G. F. Walters, "Factors in Software Quality, (RADC TR-77-369)," National Technical Information Service, Alexandria, 1977.

[19] M. Kogure and Y. Akao, "Quality Function Deployment and CWQC in Japan,” Quality Progress, Vol. 16, No. 10, 1983, pp. 25-29.

[20] V. R. Basili and H. Rombach, "Tailoring the Software Process to Project Goals and Environments," Proceedings of the 9th International Conference on Software Engineering, 1987, pp. 345-357.

[21] V. R Basili and D. M. A Weiss, "Methodology for Collecting Valid Software Engineering Data," IEEE Transactions on Software Engineering, Vol. SE-10, No. 3, 1984, pp. 728-738. doi:10.1109/TSE.1984.5010301

[22] H. D. Rombach, "Practical Benefits of Goal-Oriented Measurement," In: N. Fenton and B. Littlewood, Eds., Software Reliability and Metrics, Elsevier Science Publishing Co., London, 1991, pp. 217-235.

[23] F. V. Latum and R. V. Soligen, "Adopting GQM-Based Measurement in an Industrial Environment," IEEE Software, Vol. 15, No. 1, 1998, pp. 740 -7459.

[24] GQM Method Application, 2008. http://www-ivs.cs.uni-magdeburg.de/sw-eng/us/java/GQ $\mathrm{M} / \mathrm{link3}$

[25] I. Tumer, "Function Based Risk Assessment: Mapping Function to Likelihood," Proceedings of DETC'05, Vol. 5a, Long Beach, 24-28 September 2005, pp. 455-467. doi:10.1115/DETC2005-85053

[26] Office of the Under Secretary of Defense, "DSMC Risk Management Guide for DoD Acquisition," 2nd Edition, Defense Systems Management College Press, Fort Belvoir, 1999.

[27] A. D. Meyer and J. B. Goes, "Organizational Assimilation of Innovations: A Multilevel Contextual Analysis," Academy of Management Journal, Vol. 31, No. 4, 1988, pp. 897-923. doi:10.2307/256344

[28] J. Hirtz, R. Stone, D. McAdams, S. Szykman and K. Wood, "A Functional Basis for Engineering Design: Reconciling and Evolving Previous Efforts," Research in Engineering Design, Vol. 13, No. 2, 2002, pp. 65-82.

[29] C. Bryant, R. Stone, J. L. Greer, D. McAdams, T. Kurtoglu and M. Campbell, "A Function-Based Component Ontology for Systems Design," Proceedings of the 16th International Conference on Engineering Design, Paris, France, 28-31 August 2007, pp. 119-120.

[30] I. Tumer, R. Stone and D. Bell, "Requirements for a Failure Mode Taxonomy for Use in Conceptual Design," Proceedings of the International Conference on Engineering 
Design, Stockholm, 19-21 August 2003, pp. 109-124.

[31] R. Stone and T I. umer, "Failure Prevention through Effective Cataloguing and Utilization of Historical Failure Events,” Journal of Failure Analysis and Prevention, Vol. 8, No. 5, 2008, pp 469-481. doi:10.1007/s11668-008-9160-7

[32] J. Vucovich, R. Stone, X. F. Liu and I. Tumer, "Early Identification of Potential Software Failures Using the Software Function-Failure Design Method,” 29th Inter- national Conference on Software Engineering, Minneapolis, 20-26 May 2007.

[33] R. Patil, "Business Risk in Early Design (BRED): A Business Risk Assessment Approach,” Engineering Management Journal.

[34] E. Cudney, "Managing Lean Implementation Risks,” Proceedings of the 2009 Industrial Engineering Research Conference, Miami, 30 May-3 June 2009. 